\title{
Turbulence, Spatial Transport, and Heating of the Solar Wind
}

\author{
W. H. Matthaeus, ${ }^{1}$ G. P. Zank, ${ }^{1}$ C. W. Smith, ${ }^{1}$ and S. Oughton ${ }^{2}$ \\ ${ }^{1}$ Bartol Research Institute, University of Delaware, Newark, Delaware 19716 \\ ${ }^{2}$ Department of Mathematics, University College London, London, England
}

(Received 28 December 1998)

\begin{abstract}
A phenomenological theory describes radial evolution of plasma turbulence in the solar wind from 1 to 50 astronomical units. The theory includes a simple closure for local anisotropic magnetohydrodynamic turbulence, spatial transport, and driving by large-scale shear and pickup ions. Results compare well to plasma and magnetic field data from the Voyager 2 spacecraft, providing a basis for a concise, tractable description of turbulent energy transport in a variety of astrophysical plasmas. [S0031-9007(99)08959-0]

PACS numbers: 96.50.Ci, 52.30.-q, 52.35.Ra
\end{abstract}

Low-frequency fluctuations in the solar wind plasma represent perhaps the most extensively studied type of magnetohydrodynamic (MHD) turbulence, having been observed by spacecraft instruments for more than thirty years [1-3]. The observed turbulence displays properties expected of both hydrodynamic and MHD theories, including distinctive spectra and correlations $[3,4]$. Solar wind turbulence is a crucial element in coupling the lower corona plasma and the earth's magnetosphere, and in the transport of energetic charged particles throughout the solar-terrestrial environment. It is also a prototype for understanding stellar and galactic winds and astrophysical plasma flows in general. There has been notable progress in understanding the cascade process [5-12] that accompanies solar wind turbulence. So far, however, no single quantitative model has explained how turbulent energy flows from the largest interacting structures to the smallest dissipative scales where it is deposited as heat. In this Letter we present such a theory, based upon the dynamics of large-scale "eddies," which, controlled by a single similarity scale, drives a cascade that supplies thermal energy to the fluid plasma. The theoretical results compare well with measurements by the Voyager 2 spacecraft at heliocentric radial distances $r$ from 1 astronomical units (AU) to beyond $30 \mathrm{AU}$. This motivates the development of similar phenomenological turbulence theories for nonlinear MHD flows in a variety of astrophysical plasmas.

From the Helios and Mariner missions reaching inside $0.3 \mathrm{AU}$, to the Voyager and Pioneer explorations beyond $50 \mathrm{AU}$, spacecraft instruments have returned magnetic field data and plasma data (proton temperature, velocity, and density) that reveal the organized large-scale structure of the heliospheric plasma, along with transient mesoscale features such as coronal mass ejections and an ubiquitous but nonuniform admixture of fluctuations. Substantial fluctuation energy resides in an inferred range of spatial scales between the ion inertial scale $\left(\approx 10^{6} \mathrm{~cm}\right.$ at $\left.1 \mathrm{AU}\right)$ and the observed correlation scale $\lambda\left(\approx 6 \times 10^{11} \mathrm{~cm}\right.$ at $1 \mathrm{AU})$. The radial dependence of fluctuations in the low latitude solar wind is illustrated using Voyager 2 data in
Figs. 1-3, which portray magnetic field variance (energy density in the turbulent magnetic field), correlation length, and proton temperature, from $1 \mathrm{AU}$ to beyond $30 \mathrm{AU}$. To simultaneously explain these three data sets is a significant challenge. The main objective of this Letter is to provide such an explanation based upon turbulence theory.

Observed properties of solar wind MHD fluctuations are interpreted in two distinct ways. A distinctive velocitymagnetic field correlation is observed frequently and is suggestive of large amplitude noninteracting Alfvén waves [3]. Conversely, the wave number $(k)$ spectrum of fluctuations, having typically a $k^{-5 / 3}$ Kolmogorov form, indicates quasisteady spectral transfer and strong nonlinear couplings. This dichotomy persists when noting that the radial variation of the fluctuation energy from 1-10 AU follows the WKB $r^{-3}$ scaling rather closely (Fig. 1), suggesting again noninteracting waves [13]. However, the radial evolution of the correlation scale is inconsistent with a WKB expansion (Fig. 2). A purely wave picture also

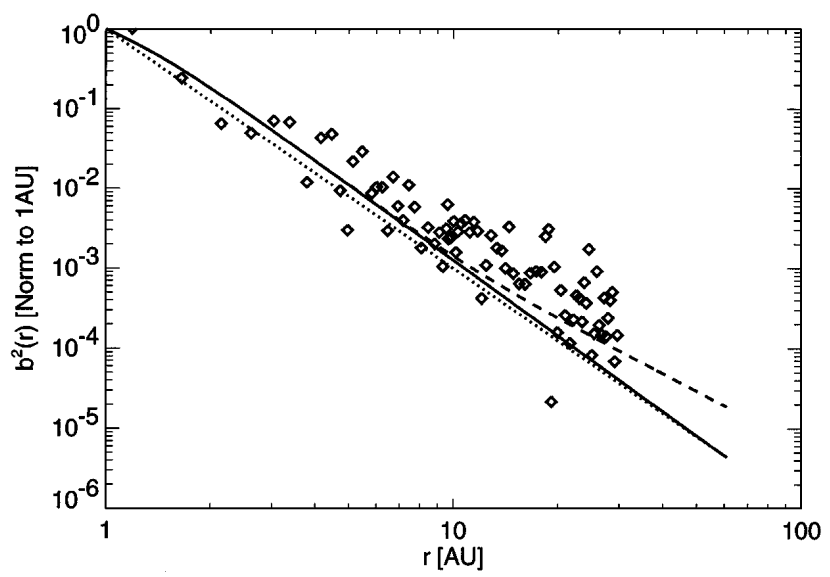

FIG. 1. Energy density of turbulence (per unit volume) estimated from 1 hour of Voyager 2 magnetic field data (symbols), from $1 \mathrm{AU}$ to about $30 \mathrm{AU}$. Theoretical solutions shown for shear driving only (solid line) and for shear plus pickup ion driving (dashed line). The dotted line is the WKB result $\sim r^{-3}$. 


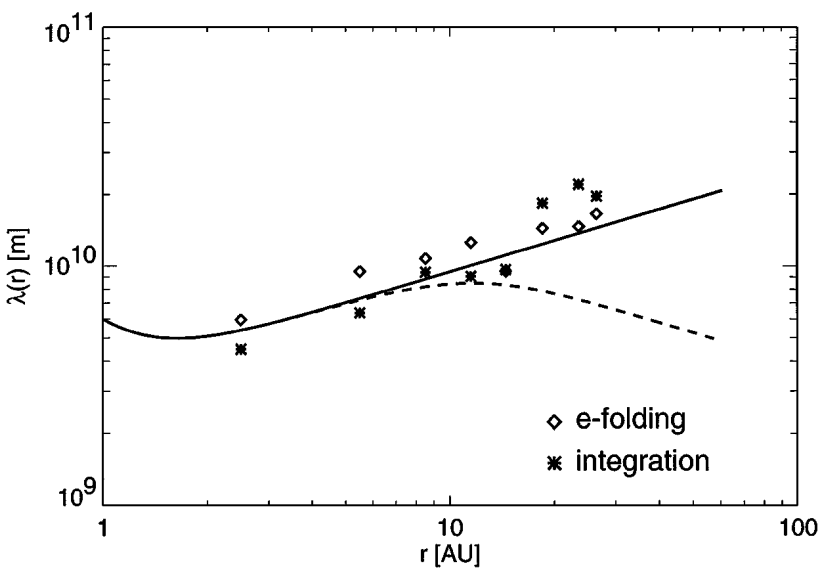

FIG. 2. Correlation scale of the normal component of magnetic field fluctuations for the same data as in Fig. 1, computing using both the integral and $e$-folding definitions (see Ref. [22]). Data are compared with the theoretical model for the Kármán similarity scale (curve styles as in Fig. 1).

cannot explain the observed (Fig. 3) highly nonadiabatic proton temperature profile [14-16]. An actively turbulent interplanetary plasma might heat the plasma and maintain a power-law inertial range, while the span of the inertial range migrates in time towards lower frequencies $[17,18]$. This corresponds, through the frozen-in flow condition [19], to an increasing correlation scale (Fig. 2), usually attributed [20] to communication of turbulent eddies to steadily increasing scales.

To develop a tractable model for the radial evolution of MHD-scale solar wind turbulence, we view the fluctuations locally as nearly incompressible [21], strongly nonlinear, homogeneous MHD turbulence [5,7]. Treatment of strong local turbulence on the same footing as spatial transport is mandated $[5,20,22]$ by the similar magnitude of the expansion time $\sim r / U$ and the eddy-turnover time $\sim \lambda / u(U$

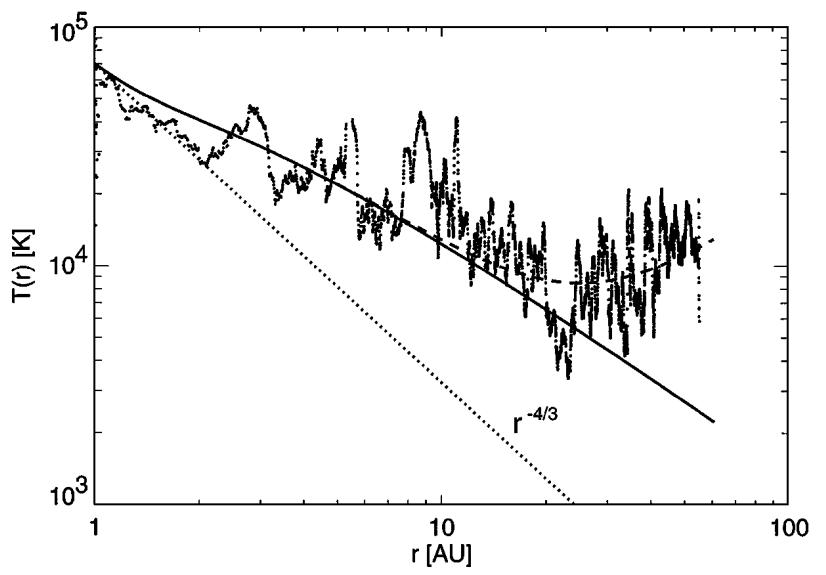

FIG. 3. Proton temperature data from the Voyager 2 plasma instrument, from $1 \mathrm{AU}$ to about $50 \mathrm{AU}$, indicating highly nonadiabatic behavior. Also shown is temperature from the theoretical model in which turbulent dissipation supplies internal energy (curve styles as in Fig. 1). denotes the large-scale flow speed, $u$ the rms turbulent velocity). To a first approximation, transport of turbulent fluctuations involves convection and propagation in prescribed large-scale plasma flow and magnetic fields. MHD turbulence transport equations are derived using an assumption of scale separation $(\lambda / r \ll 1)$, providing generalizations of WKB theory [7,23,24]. It is straightforward to derive equations for various correlation functions $[7,8]$ involving the Elsässer variables $\mathbf{z}_{ \pm}=\mathbf{v} \pm \mathbf{b} / \sqrt{4 \pi \rho}$. Here $\mathbf{v}$ is the turbulent plasma velocity and $\mathbf{b} / \sqrt{4 \pi \rho}$ is the fluctuating component of the magnetic field, normalized to Alfvén speed units ( $\rho$ is the mass density).

The present formalism does not require the full correlation functions and associated spectra. The TaylorKármán approach [25,26] describing the evolution of hydrodynamic turbulence from the perspective of the "energy-containing eddies" requires only an energy $u^{2}$ and an associated similarity length scale $\lambda$. Here we adopt such a model, based upon the self-preservation hypothesis [25,26], with adaptations appropriate to MHD [27-31]. A distinguishing feature of the MHD case, with a locally uniform mean magnetic field $\mathbf{B}_{0}$ is the appearance of anisotropy [32-36] associated with suppressed spectral transfer in the direction parallel to $\mathbf{B}_{0}$. For simplicity, we postulate that spectral transfer is of the quasi-2D or nearly "zero frequency" type, usually described by reduced MHD [21,36-38]. Accordingly, for low cross helicity ( $\mathbf{v}$ and $\mathbf{b}$ uncorrelated) the decay of incompressible turbulence energy, designated by the Elsässer variance $Z^{2}=\left\langle v^{2}+b^{2} / 4 \pi \rho\right\rangle$, takes on the hydrodynamic form

$$
\frac{d Z^{2}}{d t}=-\alpha \frac{Z^{3}}{\lambda_{\perp}}+S
$$

where the perpendicular similarity scale $\lambda_{\perp}$ may be associated, for example, with a correlation scale transverse to the mean magnetic field. Corrections to the leading order perpendicular spectral transfer implied by Eq. (1) would involve parallel spectral transfer at order $b / B_{0}[35,37,38]$. Sources of turbulent energy are represented by $S$. From $1 \mathrm{AU}$ to about $10 \mathrm{AU}$ we expect that the principle source of replenishment for small-scale turbulence is instability associated with stream shear [2,39] between regions of fast $\sim 700 \mathrm{~km} / \mathrm{s}$ wind and slow $\sim 300 \mathrm{~km} / \mathrm{s}$ wind. Equation (1) is expected to remain valid for weakly compressible MHD [21] when the turbulent Mach number, density fluctuations, and propagating compressive fluctuation are small. These conditions are reasonably well satisfied in the solar wind $[40,41]$.

In the outer heliosphere $(r>1 \mathrm{AU})$, low cross helicity $[3,40]$ and low Alfvén speed $V_{A} \ll U$ lead to considerable reduction in the complexity of the transport equations [10,11]. Combining local turbulence and spatial transport effects, the energy density evolves according to

$$
\frac{\partial Z^{2}}{\partial t}+\mathbf{U} \cdot \nabla Z^{2}+Z^{2} \nabla \cdot\left(\frac{\mathbf{U}}{2}\right)+M D=N_{Z},
$$


where $N_{Z}$ represents the right-hand side of Eq. (1). The quantity $M$ depends upon the geometry of the large-scale fields and includes large-scale compressions and shear. $D=\left\langle v^{2}-b^{2} / 4 \pi \rho\right\rangle$ is the "energy difference" of fluid and magnetic fluctuation contributions. A convenient closure is to assume that $D=\sigma_{D} Z^{2}$ for some constant $\sigma_{D}$. In the solar wind, typically $\sigma_{D} \approx \frac{1}{3}[4,41]$.

We identify the Kármán-Howarth similarity scale with the local correlation length (departing in this regard from earlier efforts [5,6]), employing the standard definition [42] $\int_{0}^{\infty} R\left(r^{\prime}, 0,0\right) d r^{\prime} \equiv L=\lambda Z^{2}$ where $R\left(r^{\prime}\right)$ is a correlation function. We can form an equation for $L$ by integration of the appropriate correlation function transport equation $[10,11,24]$ over all values of spatial separation. After some manipulation [10,11], this gives

$$
\frac{\partial L}{\partial t}+\mathbf{U} \cdot \nabla L+\left(\nabla \cdot \frac{\mathbf{U}}{2}\right)\left(1-\sigma_{D}\right) L=N_{L} .
$$

The nonlinear term $N_{L}$ associated with $L$ is specified by adopting a local conservation law, typically either $Z \lambda=$ const, or $Z^{2} \lambda=L=$ const. The former of these corresponds, for homogeneous turbulence, to $d \lambda / d t=$ $\beta Z$ with $\beta=\alpha$. The latter corresponds to $\beta=\alpha / 2$ [10].

For solar wind solutions to Eqs. (2) and (3), we assume $\mathbf{U}=\hat{\mathbf{r}} U$, with constant $U=400 \mathrm{~km} / \mathrm{s}$. The steady state equations for the energy and correlation (similarity) scale become

$$
\begin{gathered}
\frac{d Z^{2}}{d r}=-\frac{A^{\prime}}{r} Z^{2}-\frac{\alpha}{U} \frac{Z^{3}}{\lambda}+\frac{\dot{E}_{\mathrm{PI}}}{U}, \\
\frac{d \lambda}{d r}=-\frac{C^{\prime}}{r} \lambda+\frac{\beta}{U} Z-\frac{\beta}{U} \frac{\lambda}{Z^{2}} \dot{E}_{\mathrm{PI}},
\end{gathered}
$$

where we have introduced an energy supply rate $\dot{E}_{\text {PI }}$ due to pickup ions, which will be discussed presently. These are supplemented by a temperature equation in which the heat source is the energy dissipated by turbulence $[2,43]$. Thus the temperature is determined by

$$
\frac{d T}{d r}=-\frac{4}{3} \frac{T}{r}+\frac{2}{3} \frac{m_{p}}{k_{B}} \frac{\alpha}{U} \frac{Z^{3}}{\lambda} .
$$

Various constant parameters appear in Eq. (4); $A^{\prime}=A-$ $C_{\text {sh }}$, where $A$ depends upon the rotational symmetry of the fluctuations. Energy supply by shear [11] is estimated as $\dot{E}_{\text {shear }} \propto \Delta U Z^{2} / \Delta r \sim C_{\mathrm{sh}} U / r$ for shear amplitude $\Delta U$ and shear layer width $\Delta r$, thus determining the constant $C_{\text {sh. }}$. Similarly $C^{\prime}=B-A-\hat{C}_{\text {sh }}$, with $B$ an $O(1)$ geometry dependent constant [10]. Typically $\alpha \approx 1$ and $\beta=\frac{1}{2}$ to 1 , where these are the Taylor-Kármán constants associated with the local phenomenology $[10,30]$.

We have found steady solutions of Eqs. (4)-(6) for which the radial dependence of the turbulence energy $Z^{2}$, similarity length scale $\lambda$, and the temperature $T$ compare well with the corresponding quantities extracted from Voyager data. Figures 1-3 illustrate this comparison for two instructive cases. In each the boundary data at $1 \mathrm{AU}$ are $Z^{2}=250 \mathrm{~km}^{2} / \mathrm{s}^{2}, \lambda=0.04 \mathrm{AU}$, and $T=7 \times$ $10^{4} \mathrm{~K}$, with the constants chosen as $A=0.9, B=0.7$, and $\alpha=\beta=1[10,11]$.

In the first case the turbulence is shear driven with $C_{\mathrm{sh}}=\hat{C}_{\mathrm{sh}}=2$, shown using solid lines in Figs. 1-3. The shear driven model makes a reasonable prediction for the profile of turbulent energy to $20 \mathrm{AU}$ or more [11]. Focusing on Fig. 2, the upward trend of the measured correlation scale is reasonably well accounted for by the theoretical behavior of the similarity scale $\lambda$. Finally, the theoretically predicted temperature follows the Voyager proton temperatures to about $20 \mathrm{AU}$, but underestimates the large $r(\gtrsim 20 \mathrm{AU})$ observations.

The second case includes energy input due to wave excitation by pickup ions [43], a process that becomes important in the outer heliosphere. The pickup energy input scales as $\dot{E}_{\mathrm{PI}} \sim f_{D} v_{A} U n_{H} / \tau_{\text {ion }}$, where $n_{H}$ is the density of interstellar neutrals and $\tau_{\text {ion }}$ is their ionization time. The theoretical result including shear and pickup ion driving is depicted in Figs. 1-3 by dashed lines. From 1 to about 20 AU there is little difference from the first case. However for $r \geqslant 20$ AU there are notable effects associated with pickup ions. The turbulence level is slightly higher (Fig. 1), and in somewhat improved accord with the data, while the predicted similarity scale begins to decrease, an effect not seen in the Voyager data. (We suspect this artifact may be eliminated by generalizing the model to include two components - quasi-2D fluctuations and parallel propagating waves - but we defer this to future work.) On the other hand, the temperature prediction from the theoretical model with pickup ions appears to account for the Voyager proton temperatures very well (Fig. 3).

There are other interesting solutions that start with $Z^{2}$ at $1 \mathrm{AU}$ [41] higher than the $250 \mathrm{~km}^{2} / \mathrm{s}^{2}$ employed above [13]. These solutions have $Z^{2}$ up to at least $1000 \mathrm{~km}^{2} / \mathrm{s}^{2}$, lower values of the Kármán constants $\alpha$ and $\beta$ and slightly smaller 1 AU correlation scales. Since $Z^{2}$ and $\lambda$ are observed to have substantial intrinsic variability at $1 \mathrm{AU}$, we defer discussion of the range of relevant solutions to a later time.

Remarkably, the simple turbulence model outlined above accounts well for the baseline interplanetary turbulence properties observed by the Voyager 2 spacecraft from 1 AU to several tens of AU. For the first time a theory provides a concise explanation for the average behavior of key parameters that describe solar wind fluctuations. Evidently the heating of the solar wind observed beyond $20 \mathrm{AU}$ cannot be explained by shear driven turbulence alone. Driving by injection of wave energy associated with pickup ions [43] works well at a theoretical level, thus encouraging further searches for the associated waves which have so far remained observationally elusive. The present result also provides substantial support for two theoretical assertions: (1) The solar wind turbulence is dynamically active and not a passive remnant 
of coronal processes, and (2) an MHD nonlinear TaylorKármán approach to turbulent heating is defensible and at least moderately accurate, in a form that neglects Alfvén wave propagation effects [30].

The latter point is particularly relevant as the subject of the marriage of MHD spectral transfer and kinetic dissipation processes looms as an essential factor in understanding the solar wind and other important applications such as coronal heating and the galactic dynamo. The crucial point is that kinetic processes must eventually convert fluid motions into heat, but the nature of MHD transfer to smaller scales may be central in selecting which kinetic processes are influential [44]. Even though spectral transfer is controlled by the large eddies in the Taylor-Kármán picture, the present result suggests that dissipation occurs mainly through kinetic processes operating at a high perpendicular wave number.

Related theoretical models may be useful to describe transport and turbulent heating in other space and astrophysical contexts. For example, similar models may be feasible for both high latitude solar wind and for inner heliospheric conditions. It is likely that these would require reversion to a more difficult framework, including separate equations for the two Elsässer amplitudes $Z_{ \pm}^{2}$ in regions in which cross helicity and propagation effects are important. An even more challenging application is the lower solar corona, where the large-scale flow and magnetic fields are less well known but certainly governed by factors more complex than the simple radial expansion that we were able to employ here.

The research was supported by NSF (ATM-9713595, ATM-9357861), NASA (NAG5-1573, NAG5-6469), JPL Voyager Contract No. 959167, the U.K. PPARC (GRK98711), and the SDSC. We thank Voyager PIs J.D. Richardson and N.F. Ness for advice concerning MAG and PLS data.

[1] P. J. Coleman, Phys. Rev. Lett. 17, 207 (1966).

[2] P. J. Coleman, Astrophys. J. 153, 371 (1968).

[3] J. W. Belcher and L. Davis, Jr., J. Geophys. Res. 76, 3534 (1971).

[4] W.H. Matthaeus and M.L. Goldstein, J. Geophys. Res. 87, 6011 (1982).

[5] C.-Y. Tu, Z.-Y. Pu, and F.-S. Wei, J. Geophys. Res. 89, 9695 (1984).

[6] J. V. Hollweg, J. Geophys. Res. 91, 4111 (1986).

[7] Y. Zhou and W. H. Matthaeus, J. Geophys. Res. 95, 10291 (1990).

[8] E. Marsch and C.-Y. Tu, J. Plasma Phys. 41, 479 (1989).

[9] E. Marsch and C.-Y. Tu, J. Geophys. Res. 98, 21045 (1993).

[10] W. H. Matthaeus, G.P. Zank, and S. Oughton, J. Plasma Phys. 56, 659 (1996).

[11] G.P. Zank, W.H. Matthaeus, and C.W. Smith, J. Geophys. Res. 101, 17093 (1996).
[12] M.K. Verma, D.A. Roberts, and M.L. Goldstein, J. Geophys. Res. 100, 19839 (1995).

[13] D. A. Roberts, M. L. Goldstein, and L. W. Klein, J. Geophys. Res. 95, 4203 (1990).

[14] J. Freeman, Geophys. Res. Lett. 15, 88 (1988).

[15] P. R. Gazis, A. Barnes, J.D. Mihalov, and A. J. Lazarus, J. Geophys. Res. 99, 6561 (1994).

[16] J. D. Richardson, K. I. Paularena, A. J. Lazarus, and J. W. Belcher, Geophys. Res. Lett. 22, 325 (1995).

[17] L. W. Klein, W.H. Matthaeus, D. A. Roberts, and M. L. Goldstein, in Proceedings of Solar Wind 7, COSPAR Colloquia Series, edited by E. Marsch and R. Schwenn (Pergamon, New York, 1992), Vol. 3, p. 197.

[18] T. Horbury, A. Balogh, R. J. Forsyth, and E. J. Smith, Astron. Astrophys. 316, 333 (1996).

[19] J. R. Jokipii, Annu. Rev. Astron. Astrophys. 11, 1 (1973).

[20] W. H. Matthaeus and M. L. Goldstein, Phys. Rev. Lett. 57, 495 (1986).

[21] G. P. Zank and W.H. Matthaeus, J. Plasma Phys. 48, 85 (1992).

[22] W.H. Matthaeus, C.W. Smith, and S. Oughton, J. Geophys. Res. 103, 6495 (1998).

[23] E. N. Parker, Astrophys. J. 142, 1086 (1965).

[24] W.H. Matthaeus, S. Oughton, D. Pontius, and Y. Zhou, J. Geophys. Res. 99, 19267 (1994).

[25] G. I. Taylor, Proc. R. Soc. London A 151, 421 (1935).

[26] T. von Kármán and L. Howarth, Proc. R. Soc. London A 164, 192 (1938).

[27] R. H. Kraichnan, Phys. Fluids 8, 1385 (1965).

[28] P. S. Iroshnikov, Sov. Astron. 7, 566 (1964).

[29] M. Dobrowolny, A. Mangeney, and P. Veltri, Phys. Rev. Lett. 45, 144 (1980).

[30] M. Hossain et al., Phys. Fluids 7, 2886 (1995).

[31] S. Galtier, H. Politano, and A. Pouquet, Phys. Rev. Lett. 79, 2807 (1997).

[32] J. V. Shebalin, W.H. Matthaeus, and D. Montgomery, J. Plasma Phys. 29, 525 (1983).

[33] S. Oughton, E. R. Priest, and W.H. Matthaeus, J. Fluid Mech. 280, 95 (1994).

[34] S. Sridhar and P. Goldreich, Astrophys. J. 432, 612 (1994).

[35] W. H. Matthaeus, S. Oughton, S. Ghosh, and M. Hossain, Phys. Rev. Lett. 81, 2056 (1998).

[36] S. Oughton, S. Ghosh, and W.H. Matthaeus, Phys. Plasmas 5, 4235 (1998).

[37] D. C. Montgomery, Phys. Scr. T2/1, 83 (1982).

[38] R. Kinney and J.C. McWilliams, Phys. Rev. E 57, 7111 (1998).

[39] D. A. Roberts, M.L. Goldstein, W.H. Matthaeus, and S. Ghosh, J. Geophys. Res. 97, 17115 (1992).

[40] D. A. Roberts, M. L. Goldstein, L. W. Klein, and W. H. Matthaeus, J. Geophys. Res. 92, 12023 (1987).

[41] C.-Y. Tu and E. Marsch, MHD Structures, Waves and Turbulence in the Solar Wind (Kluwer Academic Publishers, Dordrecht, The Netherlands, 1995).

[42] G. K. Batchelor, The Theory of Homogeneous Turbulence (Cambridge University Press, Cambridge, England, 1970).

[43] L.L. Williams, G.P. Zank, and W.H. Matthaeus, J. Geophys. Res. 100, 17059 (1995).

[44] R. J. Leamon et al., J. Geophys. Res. 103, 4775 (1998). 